\title{
Students approaching proof through conjectures: snapshots in a classroom
}

\author{
Fulvia Furinghetti*, Federica Olivero**, Domingo Paola*** \\ *Dipartimento di matematica, Università di Genova, Italy, email: furinghe@dima.unige.it \\ **Graduate School of Education, University of Bristol, U. K., email: Fede.Olivero@bristol.ac.uk \\ ***Liceo scientifico Issel, Finale Ligure (Sv), Italy, email: paola.domingo@mail.sirio.it
}

In this paper we present a classroom experiment in which students were asked to solve an open problem. It is our premise that this kind of task fosters a 'smooth' approach to proofs that are constructed around the production and validation of conjectures. This experiment helps to construct a shared classroom culture, one of the aims of this activity. Classroom discussion and collaborative group work helps to achieve this aim. The analysis of the experiment shows that the discussion makes explicit aspects of students' thinking which would be hidden in a normal activity. We demonstrate that a deeper analysis of students' reasoning and behaviours can be better performed if lessons are video-recorded. There is a twofold pedagogic gain in using videotapes as a tool for analysing students' behaviour: firstly it offers the teacher a tool that encourages students to reflect on their reasoning; secondly, and conversely, it offers researchers a further tool for analysing students' performances.

\section{Introduction}

This study addresses the problem of introducing students to proof in mathematics. The current international debate amongst mathematics educators around this problem has shown that such introduction is a hard task for educators, for many different reasons, see [1], [2], [3], [4]. One of the difficulties consists of the fact that, as Moore points out in the case of the United States, "the transition to proof is abrupt" and this "abrupt transition to proof is a source of difficulty for many students, even for those who have done superior work with ease in their lower-level mathematics courses" [5, p.249]. The same problem is encountered in many different countries (including ours) and at different school levels. Another factor which makes the transition to proof so difficult is the typical classroom environment, described in [6, p.32] as follows:

In the classroom, the teacher and the textbook are the authority, and mathematics is not a subject to be created or explored. In school the truth is given in the teacher's explanations and the answer book; there is no zig-zag between conjectures and arguments for their validity, and one could hardly imagine hearing the words maybe or perhaps in a lesson.

Within this environment, tasks concerning proofs are often presented in the form "prove that...", where the statement to be proved is already provided to students. On the contrary, we think that presenting a task in a form that fosters dynamic exploration of statements might support students in the process towards proof. The classroom style we advocate to promote the transition to proof is conveniently described by a metaphor of Pollak, quoted in [6, pp.41-42]: "to move around in mathematical territory in a flexible manner", that is to say to do a kind of "cross-country" mathematics, instead of "walking on a path that is carefully laid out through the woods". Sharing this teaching perspective might make the way of doing mathematics in the classroom closer to the way mathematicians do. 
The literature in mathematics education reports on various attempts to create a learning environment which fosters this idea. In line with the basic assumption found in [7], according to which cultural and social processes are integral to mathematical activity, we feel that both the problems to be given to students and the way in which students are required to participate in the classroom life may affect students' transition to proof. The paper elaborates this claim, by reporting on a classroom experiment (conducted in an Italian context) in which students were asked to tackle an open problem by working in groups and engaging in discussion within groups and among groups. We will outline the key stages of the experiment and only some episodes of the classroom life (the 'snapshots' of the title) will be presented in more details. We think that this structure of the paper allows the readers to participate in our experiment and to produce their own explanations of the observed behaviours to be compared with the ones we give as a comment to the snapshots.

\section{Theoretical background}

\section{Open problems}

Of the many existing classifications of problems, the most relevant for our purpose is the one referring to open compared to closed problems. Broadly speaking, the 'concept of open problem' can be defined as follows, see [8, p.1]

In a closed problem, both the starting and the goal situation are closed, i.e. they are exactly explained in the task. If the starting situation and/ or the goal situation are both open (i.e. not closed), we have an open problem.

Several types of activities have been classified under the label of open problems, including investigations, problem posing, real-life situations, projects, problems without questions, problem solving, see [8]. Since 1980 the idea of open approaches to mathematics spread all over the world and research on their potentialities is now very vivid. In the United States the Curriculum and Evaluation Standards for School Mathematics of the National Council of Teachers of Mathematics (1989) included problem solving as a method to improve the level of mathematics teaching. Problem solving has been defined as "the process of applying previously acquired knowledge to new and unfamiliar situations" [9, p.471].

In England, in addition to the concept of problem solving, investigation and investigational work have been included in the National Curriculum since 1989 and they became officially legitimised by the Cockroft report in 1982. Evans in [10] explains the difference between problem solving and investigations as follows: problem solving is convergent action where pupils have to find a solution for a certain problem, while investigation is more divergent and here pupils are encouraged to think of alternative strategies, to consider what would happen if a certain route is followed or to look at whether different approaches will produce different results. In 1988 investigations entered in the examination system in the UK via the General Certificate of Secondary Education (GCSE), which required an assessed element of coursework involving practically based studies or investigations [11] and referred to as project work. In project work students are given a situation and then they need to develop their own ideas, formulate and extend problems, and use their mathematics. As Boaler puts it, the approach "is based on the philosophy that students should encounter a need to use mathematics in situations that are realistic and meaningful to them" [12, 
p.49]. The projects usually last 2 or 3 weeks and at the end students are required to write a report describing their work and their mathematical activity. A research on the potentialities of such approach compared to the traditional teaching is presented in [12].

Since the 1970s Australia has generally followed the USA model of problems solving but trying all the time to find a compromise between the American and the British approach [8]. The VCE (Victorian Certificate of Education) made compulsory for all students to spend at least $20 \%$ of their mathematics time on independent mathematics projects and at least $20 \%$ on problem solving and modelling, which is defined as the creative application of mathematical skills and knowledge to solve problems in unfamiliar situations, including real-life situations [13]. The essential features of problem solving are considered to be

a certain degree of unfamiliarity, a freedom for students to follow their own paths to a solution, rather than following a routine or procedure set by the teacher. When the application of mathematics to a real situation is involved, problem solving tasks should generally require the formulating of a suitable mathematical model and interpreting and evaluating results. [13, p.9]

In the U.K. and Australia the fact that open problems are part of the curriculum and of the examination system has had a side-effect: teachers tend to look for strategies and routines which students can be trained to carry out in order to satisfy the coursework requirements [14]; in a sense they close the problems.

In France open problems have not been officially included in the curriculum yet. The characterisation of open problems given by Arsac et al. in [15] is aimed at fostering a teaching and learning activity which allows students to 'do' mathematics:

- The statement is short, so that it can be easily understood, it fosters discovery and all students are able to start the solution process;

- The statement does not suggest the method of solution, nor the solution itself, but it creates a situation stimulating the production of conjectures;

- The problem is set in a conceptual domain which students are familiar with. Thus students are able to master the situation rather quickly and to get involved in attempts of conjecturing, planning solution paths and finding counter-examples in a reasonable time.

Provided this general background on open problems ${ }^{1}$, many studies support the validity of open problems to stimulate productive thinking and to raise motivations to prove. The feeling of uncertainty created by the production of a conjecture is combined with the need of communicating and defending it within a community, see [16]. In [17] it is shown that in an open mathematical situation students can write varied and original responses to a given task and thus it is fostered the development of students' creativity in mathematics. However there is the danger of closing up open problems, as it has happened in the U.K., where the mathematical processes of specialising and generalising, that are characteristic of the transition to proof and can be fostered by investigations, have been transformed into the sequence

try some simple cases

make a table

guess a formula

write it up. [14, p.15] 
Moreover the focus has been mainly on making students begin to investigate, without asking them to explain to others why their conjectures hold, so that the transition to proof has not really been stimulated.

In Italy we can say that the use of open problems was originated with particular respect to the issue of the transition to proof and it is not yet widespread in schools. In the official national curriculum for mathematics suggestions in this direction are almost non existent. Studies such as [1], [18], and [19] illustrate attempts of overcoming the difficulties of the transition to proof through the use of activities which encompass: the production of conjectures and the construction of proofs or refutations of the produced conjectures. Our assumption is that proving a result that has been discovered and validated by the students themselves is more meaningful to them than proving something they are given.

This kind of activities can be classified under the label of open problems, within the broad definition given in [15]. More specifically, the problems we will refer to in the paper have the following characteristics:

- the statement is short, and does not suggest any particular solution methods or the solution itself. It usually consists of a simple description of a configuration and a request of giving statements about relationships between elements of the configuration or properties of the configuration;

- the questions are expressed in the form "Which configuration does [...] assume when [...]?", "Which relationship can you find between [...]?", "What kind of figure can [...] be transformed into?".

In general, presenting a problem in an open form hinders the implementation of a procedure or routine that has to be remembered by heart. On the contrary, students have to make their own decisions in choosing a solution path. An open problem allows freedom in producing conjectures. It requires students to pose questions rather than only to answer predetermined ones. The process of solution becomes as important as the solution itself; the attention is not only on 'producing the correct result', but also on 'how to produce a result'.

\section{Group work}

Together with the type of problem, the way students work in the classroom proves relevant to the issue of the transition to proof. When speaking about open problems, most authors underline the value of mathematical discussions in the development of pupils' mathematical thinking [8]. The role of collaborative problem solving and peer interaction in small groups has become an important area of research in mathematics education, see [20], [21], [22], [23]. Such interaction is seen to provide support for the construction of mathematical meaning by pupils, since it allows more time for pupils to talk and exchange ideas. However this way of working in not unproblematic, see [20]. Kieran and Dreyfus in [23] report on different types of interaction which occurred during a problem solving activity, with a focus on the difficulties involved in "entering one another's universe of thought" [23, p.112]. The role of the teacher with respect to the group interaction reveals fundamental as well, see [20]. One of the features at stake is how students engage in and perceive their group work. We are particularly interested in the idea of collaborative group work, characterised in [24, p.282] as follows:

collaborative group work, in which students work jointly on the same problem at all times, is linked with ideas such as situated cognition, scaffolding, and the zone of proximal 
development. As Damon and Phelps (1989) ${ }^{2}$ make clear, this is fundamentally different from co-operative learning, which refers to distinct principles and practices such as specific role assignments in a group, and goal-related accountability of both individuals and the group.

Doing collaborative group work means for students to be aware of the fact that everyone can and should contribute to the solution of the problem, and that sharing and comparing strategies and ideas is much more productive than working alone.

Small groups work on open problems and classroom discussion are modalities of working which are regularly used by the teacher who conducted this classroom experiment. They are not limited to geometry, but they are widely used also in other mathematical areas. The main issue related to this mode of working is to identify activities and learning environments in which students can be supported in the approach to theoretical thinking (the transition to proof sits within this broader framework). Introducing students to theoretical thinking means moving on from naive empiricism and from the tendency to gather conclusions or to justify conjectures on the basis of the observation of a limited number of cases or of particular situations. The term 'moving on from' underlines the fact that the starting point is observation and exploration. Therefore the activities and the tools teachers provide students with should have features that foster exploration, observation, discovery of patterns; the produced observations and conjectures should then be analysed, accepted or refuted by students through group or classroom discussion orchestrated by the teacher. The transition to theoretical thinking happens when students ask why a certain conjecture is true, after being convinced that it is true; this requires finding reasons within a more or less defined theory. This kind of approach to mathematics is similar to what happens with experimental disciplines. The analogy relies on the fact that, in both cases, the starting point is the perceptive and empirical level: regularities are discovered through exploration and convincing arguments are provided in order to validate or refute these conjectures. After that, both in mathematics and in experimental sciences, ones asks why these conjectures, which have already been validated, are true: this requires reasoning within a theory and producing arguments which must be valid within that theory. In this ongoing transition from empiricism to theory, students make meanings for the mathematical objects they use, through a never ending dialectic between personal and institutional meanings for those objects, see [25].

\section{The classroom experiment}

One of the authors (D. P.) is a Secondary School teacher. His methodology of working in the classroom involves the regular use of open problems with students. The classroom experiment we discuss in this paper was one of his first attempts to use open problems in this third year classroom (16-17 year old students) of a Liceo Scientifico ${ }^{3}$. There were 15 students in the classroom; the scheduled time was five mathematics classes per week. The students turned to be keen on doing mathematics and their previous knowledge on the subject (concerning algebra and geometry) was satisfactory. They were not used to working in groups, however most of them were quite enthusiastic about this new way of doing mathematics, both the ones who found some difficulties at the beginning and the ones who adjusted well to this novelty.

The problem given to the students is taken from $[15, \mathrm{p} .48]$ : 
You are given a right-angled triangle $A B C, A B$ being the hypotenuse. Take a point $P$ on $A B$. Draw the parallel lines to $A C$ and $B C$ through $P$. Name $H$ and $K$ the points of intersection with $A C$ and $B C$ respectively. For which position of $P$ does the line $H K$ have minimum length?

Figure 1, which represents the problem, was not given to the students.

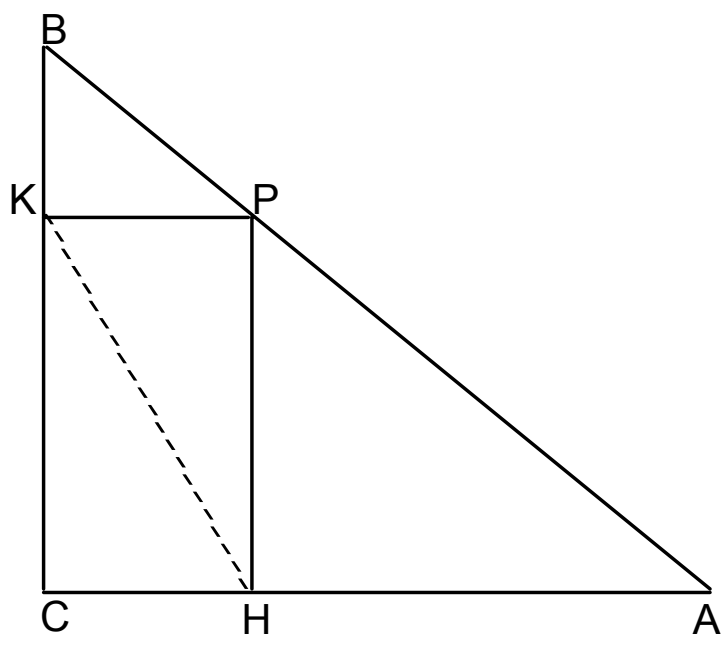

Fig.1. Illustration of the problem

The students are required to produce conjectures, which then need to be proven. This problem is open according to our definition because, as the didactic analysis in [15] shows, allows a variety of solving strategies, which are not suggested in the statement. Thus students may exploit knowledge and techniques previously acquired, in particular in synthetic and analytic geometry.

The classroom activity was organised in two parts. First, students were divided in groups (three students per group) and were given one hour to explore and solve the problem. During this part, the teacher intervened only if students required his help, making sure to foster discussion among students and not to close the debate. In the second part one student from each group presented the group process and solution in front of the whole class; a classroom discussion orchestrated by the teacher allowed the students to compare all the strategies they had produced. The whole experiment was video-recorded and watched in the following classes.

The groups were formed according to the following criteria:

- avoiding the presence of very extrovert and very introvert students in the same group, in order to foster group discussion;

- putting in the same group students who are happy to work together and used to do it, in order to provide a friendly atmosphere;

- providing the same level of students' achievement within a group, in order to avoid very good students providing the solution too quickly and to let everyone take part in the process.

The whole experiment was videotaped and the teacher wrote fieldnotes. The analysis we will present in the following sections is based on the video, the notes taken during the session by the teacher and the students' work on paper. 


\section{The students' group work}

Our experiment shows a variety of solving strategies and ways of interacting (within groups, among groups and with the teacher). In this section we focus on the description and analysis of the students' group work, with respect to modes of interaction and solving strategies. As a general remark, we point out that, since it was one of the first times in which these students were exposed to this type of work, they were quite embarrassed, especially for the presence of the video camera. Moreover, the room was suitable to traditional sessions, but not to ours; in some moments this made it difficult to carry out our activity. In this paper we confine ourselves to consider students' behaviour; nevertheless we point out that the role of the teacher was very important in the development of the students' group work and would deserve a specific study. On the one hand, he had to take responsibility for intervening at the right moment and to use efficiently the students' voices. On the other hand, he had to avoid too a massive participation in the discussion.

\section{-• Group 1: Camillo, Silvia, Ivan}

The three students sit together but work on separate papers. They feel the need of working on their own first, and they communicate only in case they need help. Probably, they do not believe in the richness of group work compared to individual work.

Regarding the solution to the problem, Camillo, Silvia and Ivan exploit many different strategies. They use ruler and measures; they work a lot with the equivalence of areas. They do not use analytical geometry at all. They are not able to provide a solution by the end of the group session.

\section{•. Group 2: Marta, Tatiana, Elisa}

This group shows a totally different modality of working. Marta, Tatiana and Elisa tackle the problem together. They write on one sheet of paper, they all speak in turns, paying attention to what the others say. This group can be considered a collaborative one.

Elisa, Marta and Tatiana start with considerations in synthetic geometry: Elisa draws the diagonal $P C$ of the rectangle $C K P H$. However this path is abandoned in favour of other ideas, mainly exploiting analytical geometry. Only at the end, they go back to the initial idea; the solution the group provides consists of realising that $H K$ equals $P C$, because diagonals of the same rectangle, and using the fact that the distance of a point from a line is the length of the segment joining the point to a point of the line and having minimum length.

In this case we might think that such a strong collaboration caused a delay in the final solution, due to the very soon abandonment of Elisa's idea. Perhaps, in a competitive group Elisa would have pursued her strategy and solved the problem more quickly.

\section{•• Group 3: Mauro, Luca, Claudio}

Mauro, Luca and Claudio find collaboration extremely difficult. Luca and Mauro work on their own and come to two different solutions. Eventually, Claudio, who seems wanting to collaborate, shows the equivalence of the two solutions. This is a group that, according to [26], would be characterised as a 'pseudo-learning' group: the students work together but they are not interested in doing this. They are very competitive, they hide useful information and they do not trust their 
colleagues. Such a group might be of no help at all to students; it could even diminish the potentiality of individuals.

In solving the problem the students begin by considering particular cases and using measures in a dynamic way. Luca uses the ruler and moves it in order to show how the length of the segments varies. This may be considered an actual exploratory behaviour, which starts from concrete/experimental situations. Mauro suggests the final solution they come up with. He considers a pencil of circles centred in $C$ and going through $P$. The circle whose radius is minimum is the circle tangent to the segment $A B$; therefore $H K$ has minimum length when $P$ is the point of contact (point of tangency) between $A B$ and the circle centred in $C$. This idea came from applying Pythagoras's theorem to the triangle $P H K$, in a system of co-ordinates centred in $C$, which produced the formula

$$
\sqrt{x^{2}+y^{2}}=d .
$$

Figure 2 shows some drawings taken from the papers this group handed in. 


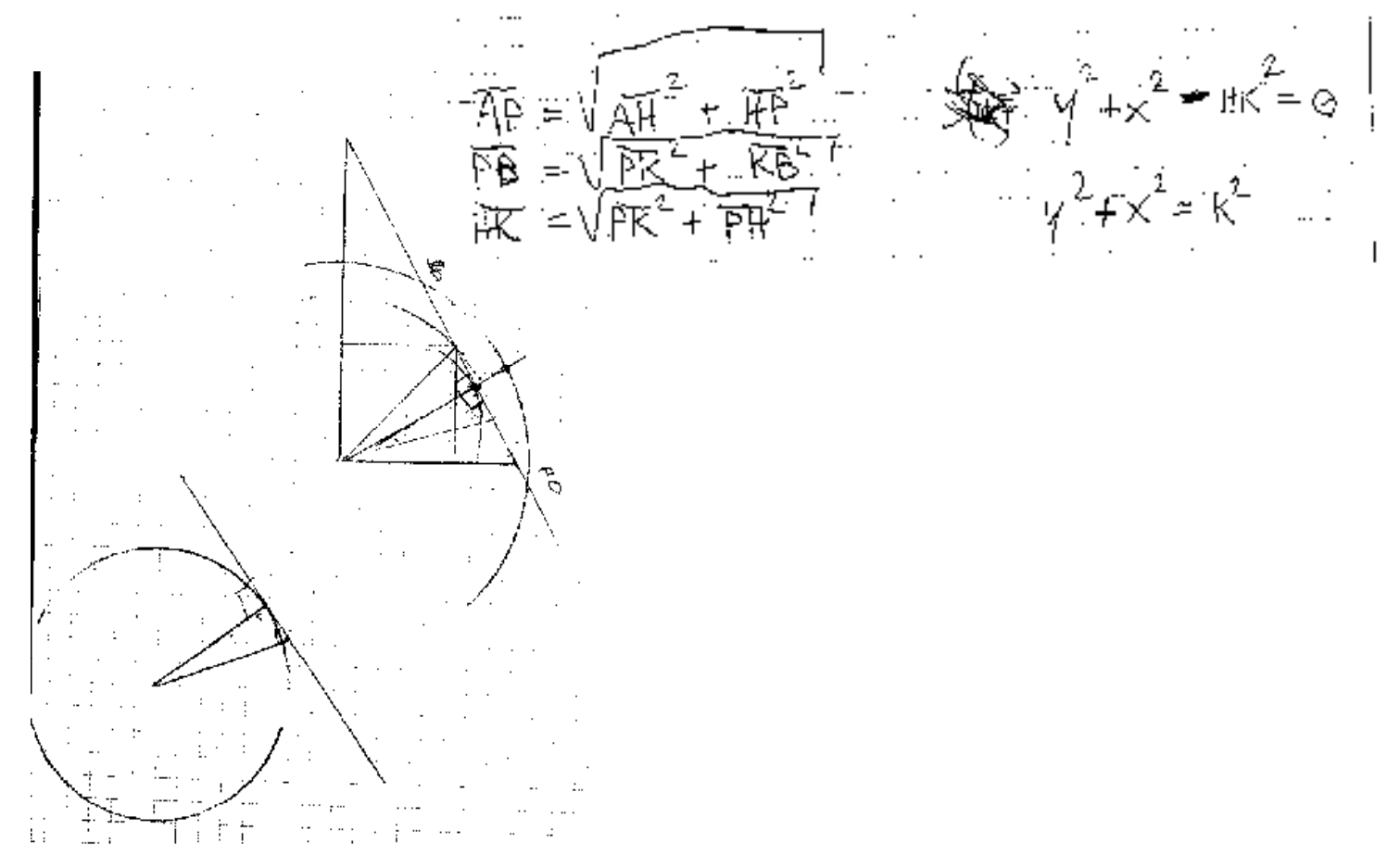

Fig.2. Mauro's protocol

This group shows a dynamic approach to the problem, which could be interpreted as transformational reasoning. This type of reasoning is described in [27, p.201] as follows: 
transformational reasoning is the mental or physical enactment of an operation or set of operations on an object or set of objects that allows one to envision the transformations that these objects undergo and the set of results of these operations. Central to transformational reasoning is the ability to consider, not a static state, but a dynamic process by which a new state or a continuum of states are generated.

We remind the reader that these students have been using the dynamic geometric software CabriGéomètre $^{4}$ and it seems that they are able to transfer the skills and the different way of looking at problems acquired working in Cabri to other situations. For example, as we have observed in other studies, a typical feature, which is borrowed from the work in Cabri is the use of circles, which are not so much exploited when working with paper and pencil. The students also transfer from Cabri the dynamic way of looking at the problem; this way provides different models of a given situation, which may foster generalisation.

\section{•.• Group 4: Vittorio, Greta, Alessia}

This group shows to have realised collaborative work. Our observations, especially those performed by watching the videotaped session, reveal the great potentialities of the discussion in small groups: for example, Alessia uses her hands a lot, in order to tell the others what she is thinking. Individual problem solving would not allow such an activity, therefore many communication and listening strategies, which play an important role in this phase of the solution process, would be lost.

As far as the solution is concerned, the students begin with synthetic geometry first, and then they switch to analytical geometry and explore extreme cases. They perform dynamic explorations, especially Alessia, who moves her hands on the figure in order to show what happens when the point $P$ moves on $A B$. In our opinion, these dynamic explorations might be fostered by the work in small groups. At the end they provide the same solution as Group 2.

\section{•. Group 5: Claudia, Monica, Michela}

The students seem to collaborate. However a deeper analysis of their work suggests that this group is, according to [26], a 'traditional learning group'. Students agree to work together, but they are not organised in the division of tasks. They are mainly concerned of the fact that they will be assessed as individuals and thus they are not interested in a learning exchange with their colleagues. Their way of dealing with the problem would probably be the same if they were working on their own.

Claudia, Monica and Michela are not deeply engaged with the new kind of work they are required to do (conjecturing). Their desk is covered with traditional learning tools, e.g. books, notebooks, ruler, compass, calculator. These students seem to rely on these tools in order to find a solution. They spend a lot of time in browsing books in order to find any useful information related to the given task. At some point, Michela says "do you remember that time we studied...", referring to a topic studied the previous year. They do not follow a precise strategy and they can't come up with a meaningful solution by the end of the session. 


\section{Zoom on the case of Marta: Euclid vs. Descartes in students' strategies}

After the first phase of group work described in paragraph 3, the teacher raised the classroom discussion. All groups were asked to present their solution strategies to the whole class; different aspects emerged from this. We discuss a case which in our perspective is very significant, since it brings to light an important feature connected to the activity of proving in geometry. The classroom discussion was a key point for this feature to emerge and video-recording the session was very useful for being able to make further reflections on the case. In the following, we quote some episodes of the discussion, which in our opinion show important elements of students' reasoning to be studied. The crucial point is Marta's claim that with analytical geometry "we can find exactly were the point is", while in Euclidean geometry "we have to know measures". At this point all the students participated in the debate. The issue raised was so important that the teacher came back again to this point a year later, watching again the videotape of this session with the students.

Marta: "So... er... we considered a generic right angled triangle. Then the first thing we noticed was that $P K C H$ stays a rectangle, for any position of $P$ on $A B$ "

$[\ldots]$

Marta: "Then we realised that $K H$ equals $C P$, since they are both diagonals of the same rectangle. Therefore we can consider $C P$ instead of $K H$. Then $P \ldots$ well... We said that the shortest distance through... I mean from a point to a segment, was that segment perpendicular to the given line, in this case to the hypotenuse and passing through $C$. Therefore $P$ must be the foot of the altitude related to the hypotenuse, because it's that segment through $C$ perpendicular to $A \mathrm{~B}$.

$[\ldots]$

Marta: "While using Euclidean geometry we can determine $P$ with a figure, using analytic geometry we solve a system with the line through $C$ perpendicular to $A B$ and we can find the coordinates of the point"

Teacher: "Do you agree with the fact that using Euclidean geometry we can determine $P$ with a figure?"

Ivan: "We draw the line through $C$ perpendicular to $A B \ldots$..

Michela: "We draw the altitude. Then $P C$ is exactly the altitude, so I can draw it... "

Teacher: "OK. But what do you mean when you say 'with a figure'? Do you mean that in Euclidean geometry I can determine $P$ only with a figure?"

The teacher is trying to stress the crucial point in order to make it clear for all students. In the video we hear Camillo stating that "first comes Euclidean geometry and then analytical geometry, without Euclidean geometry, analytical geometry wouldn't exist: however analytic geometry is more precise".

At that time the teacher did not make further comments on this issue, but he realised that at that moment students were lacking the idea of 'theory': Euclidean geometry was considered made of figures only and not based on axioms, definitions, theorems.

The classroom discussion revealed itself very important to plan further work around proof. The activities that followed this first open problem with discussion (construction problems in Cabri aimed at developing the sense of the theory which lays behind the drawings produced on the screen) were suggested to the teacher by the difficulties encountered by students in understanding the existence of a 'theory' underlying their geometrical practices. Later on, the teacher showed 
again the bit of the video in which Marta is saying the words quoted above in order to go back to the issue of the geometrical theory behind figures.

\section{Our hypotheses on Marta's behaviour}

The elements mentioned before (classroom discussion and analysis of videotapes) revealed themselves also very important to reflect on the students' ways of thinking. One of the important features we were able to single out thanks to the discussion and the videotape is the duality between analytical and synthetic geometry (see 'Zoom on the case of Marta'). This provides an example of how this kind of work in classroom can be fruitful for researchers, as well as for teachers. We consider the case of Marta, because we were struck by the way she accepts results if they come from analytical geometry, while she does not trust those coming from Euclidean geometry. The problematique we deal with is linked to the duality between visualisation and analytical thinking, as discussed in [28]. The characterisations given in [28] are as follows:

Definition: Visualisation is an act in which an individual establishes a strong connection between an internal construct and something to which access is gained through the senses. Such a connection can be made in either of two directions. An act of visualisation may consist of any mental construction of objects or processes that an individual associates with objects or events perceived by her or him external. Alternatively, an act of visualisation may consist of the construction, on some external medium such as paper, chalkboard or computer screen, of objects or events that the individual identifies with object(s) or process(es) in her or his mind. (p.441)

and

Our use of analysis may be better illustrated by the biologist who analyses the nature of a plant through decomposing it into its parts, as well as thinking about the relationships among those parts and synthesising them into various other wholes such as leaves, flowers, and seeds. Thus, we include the naming of parts in our view of analysis, but we also include intellectualising about problems through thinking about relationships among those parts and synthesising them into various new wholes.

Definition: An act of analysis or analytic thinking [...] is any mental manipulation of objects or processes with or without the aid of symbols. (p.442)

As we will see, the particular context of proving in Euclidean geometry slightly changes the meaning of these two terms: in our case, visualisation mainly means geometrical drawings or geometrical figures, while analytical thinking is linked to the analytical method, both in the sense of analysis as opposed to synthesis, see [29], and in the sense of analytical geometry. In our frame there is not an a priori hierarchy related to the importance of these two components (visual and analytical) of knowledge. Instead, we fully agree with the idea expressed in [28] that learning is a process developed through an interactive relationship between visual and analytical thinking, which may become essential within the context of the transition to proof.

How does this interaction work in the case of Marta? We tried to provide an explanation for her behaviour and to construct hypotheses to be further investigated. These hypotheses are not contrasting each other; they, indeed, can be integrated to develop a more complete explanation of the case in question. 
First hypothesis. Dealing either with figures or with letters seems to show similar patterns. Both these entities are linked to particular contexts. The visual context is very rich in geometry: when dealing with figures we can consider shape, measure, colour, position, etc. In other experiments, [30] for one, we found that rich contexts act as an obstacle. When working with algebra, students meet other types of problems: in this case the context may be too poor and there may be a lack of information. Nevertheless in algebra, symbols (for example, letters) are forms of representation close to the 'generic'. The importance of the generic in the mathematical activity is widely discussed in [31], and is a key issue as far as proof is concerned. On the ground of these considerations, we think that the visual context requires efficient representations suitable for students to grasp the meaning, which stands behind the drawing. For this reason, after this activity the teacher made students work with Cabri, with the aim of exploiting the dynamic features of this geometric software to make explicit to students both the operational and the linguistic level of drawings. Cabri generates a quantity of examples of figures, and, as a consequence, generates mental images which help to approach the generic.

Second hypothesis. Reasoning with geometric figures implies using the synthetic method, while analytical thinking relies on the method of analysis. Here the word 'analysis' does not refer to the part of mathematics known with this name, but to the method of solving problems by starting from a statement to be proven and searching if it is a consequence of other statements [29]. It is well known that this method (which has old historical roots, see [32], and has recently been rediscovered as the top-down method) is a powerful way of solving problems and proving theorems. As a matter of fact, this method was used by mathematicians to prove a lot of theorems; only after this first phase of analysis, theorems were then presented in the synthetic form (where statements follow from assumptions such as axioms and definitions).

Third hypothesis. Marta is not able to make a clear distinction between processes and results of processes: the point $\mathrm{P}$ is well defined when a process to find it is discovered, while drawing is only a means of representing this process. Since the process of identification of a point remains hidden in Marta's eyes, she is not able to understand that the precision of a drawing has no role in deciding the correctness of the discovered result. This third hypothesis is strongly supported by the behaviour both of Marta and of the students of Group 5. They rely in a rote-like way of working based on reproducing what an authority (the teacher, the book) has already shown, instead of developing strategies by themselves.

\section{Summary and implications}

In our opinion the study reported in this paper offers elements of reflection both to educators interested in introducing innovations in the mathematics classroom and to the ones interested in studying teaching and learning processes. The summary of the main findings allows us to draw some conclusions and outline future developments.

The work in the classroom and our analysis have stressed the importance of videotaping sessions in order to build a classroom story students are aware of. By videotaping a session and watching the video with the students, we might reduce the risk that significant moments of the classroom life be neglected or forgotten by students over time. The teacher himself may grasp voices, which would remain hidden in a normal classroom and may exploit these recovered voices 
to discuss problematic issues. This fact also happened when we looked at the video as mathematics education researchers.

The use of the videotape allowed the teacher to underline certain points many times after the discussion actually took place and to stress the difficulties encountered; it proved itself a powerful didactic tool. As a matter of fact the possibility of watching a session from the outside, stopping the video when you want, choosing the bits to be shown, going backwards and forwards is a powerful tool which allows to bring to light the most significant 'voices' in the classroom. When the teacher refers to a situation (teacher's explanation, a student's intervention, an exercise,...) which has been not only experienced, but also watched, analysed and criticised by the students themselves, the impact on students can be very different from reminding them a situation which has to be reconstructed only through memory, and that they probably have forgotten. The analysis of students' behaviour through the videotape suggested the teacher to devote more time to the use of dynamic geometric software to emphasise the 'theoretical aspects' of drawing and to make students acquire awareness of the theory behind their drawings. The teacher had to take into account the students' rooted beliefs on the nature of geometry. When dealing with habits, which are deeply rooted in students' mind, it is necessary to go back again to the point. In Marta's case this was possible thanks to the video.

Regarding open problems, we have seen that the use of open problems and classroom discussion helped students to produce strategies and, because of the need of communicating them to their colleagues in the classroom, to make them explicit. This allowed the teacher to plan future work starting from students' real needs as emerged from our multi-faceted classroom observation. At the same time, making strategies explicit is also a powerful research tool that allows access to students' learning processes.

A recent paper has drawn our attention towards the issue of creative thinking, whose key aspects are "the ability to overcome fixations in mathematical problem-solving and the ability for divergent production within the mathematical situation", see [33, p.187] ${ }^{5}$. In Imai's paper divergent thinking is characterised in terms of the following features:

fluency, shown by the production of many ideas in a short time; flexibility, shown by the students varying the approach or suggesting a variety of methods; originality, which is the student trying novel or unusual approaches; elaboration, shown by extending or improving of methods; and sensitivity, shown by the student criticising standard methods constructively. (ibid.)

We feel that the behaviour of Group 3 is an evidence of the potentialities of open problems in stimulating divergent thinking. Some ideas emerged that would not have been present in a normal session.

What remains under discussion and needs further adjustments is the realisation of the collaborative group work style. We stated that one of the aims of our activity in the classroom was to create a shared classroom culture and we considered collaborative group work to be an effective means towards our aim. Three out of five groups seem not to accept this form of interaction. Two points of view in the classroom (the students' and the teacher's) have to come to terms: the goal of the students is to solve the problem; the goal of the teacher is to create an environment in which all students have the possibility to solve the problem. The reaction of our students does not match the conclusions found in [24], but we have to acknowledge that there are some differences between the 
two experiments. In both cases the teacher carrying out the experiment was strongly and coherently convinced of the efficacy of collaborative group work, but definitely our students worked for much less time than the students considered in [24]. Time plays an important role in introducing students to the new style of working, which cannot be taken for granted in the classroom, but has to be taught and acquired through repeated experiences.

The following passage found in [35, p.474] provided us with suggestions aimed to find out which kind of intervention has to be performed to succeed in dealing with collaborative group work:

the suggestion that students can be left to their own devices to construct the mathematical ways of knowing compatible with those of wider society is a contradiction in terms.

To face the problem of changing the classroom style of work we feel the need to enlarge our considerations to issues concerning social and socio-mathematical norms in the classroom, in line with the discussion presented in [34] and [35]. In these papers the authors analyse how students behave when they have to participate in a new teaching project. In their opinion students' participation is actual and efficient

only to the extent that they [students] have constructed personal ways of judging that enable them to know in action both when it is appropriate to make a mathematical contribution and what constitutes an acceptable mathematical contribution. This requires, among other things, that students can judge what counts as a different solution, an insightful solution, an efficient solution, and an acceptable explanation. But [...] these are the types of judgements that the teacher and students negotiate when establishing sociomathematical norms that characterise an inquiry mathematical tradition. In the process, students construct specifically mathematical beliefs and values that help form their judgements. [35, p.473]

In [34], Cobb and Yackel point out that there is a reflexive relationship between the constitution of the classroom social norms, that are consistent with an inquiry approach to instruction, and the students' reorganisation of their beliefs about their role in the classroom, the teacher's role in the classroom, and about what constitutes mathematical activity. A consequence of this is that not only each one contributes to the constitution of the other, but also that one does not exist without the other. Further, it means that a change in beliefs occurs concomitantly with the constitution of norms. We think that the work in collaborative groups would have been carried out more efficiently with a preliminary work on students' beliefs.

The interlacement of social and sociomathematical norms and students' beliefs in the acceptance of collaborative group work emerged in our groups. We have seen that the students of Group 5 care of the individual assessment more than of the solution of the task. This fact may have affected their negative reaction to the new way of working. The belief underlying this behaviour is mainly linked to the role ascribed to instruction in individuals' life.

Other types of beliefs affected the acceptance of collaborative group work in the considered classroom. As pointed out by McLeod [36], [37] we have to take into account that beliefs encompass cognitive as well affective aspects. Both aspects influence the students' responses to input given by the teacher. We may interpret the behaviours of the different groups as an evidence of the impact of affective factors. In Group 2 the low self confidence of Elisa provoked the abandon of her proposed (correct) strategy. In Group 3 the theory of success espoused by students is based 
on individual rather than on social issues. In Group 5 the strong trust in external authority (traditional learning tools) is hindering autonomous thinking.

Eventually we may say that the main findings about the key elements characterising our experiment of introduction to proof - i.e. tools of observation, open problems, collaborative group work - are only partially conclusive. In particular, collaborative group work needs further investigation and analysis both in classroom and in educational research. Nevertheless our feeling is that our experiment shows that all these elements are essential components in the students' approach to proof that we have advocated at the beginning.

\footnotetext{
${ }^{1}$ A detailed discussion of the different approaches in the different countries is not the aim of this paper.

${ }^{2}$ From references in [8]: Damon, W. and Phelps, E., 1989, Int. J. Educ. Res., 13, 9-19.

${ }^{3}$ Liceo Scientifico (Scientific Lyceum) is a type of Italian secondary school with a scientific orientation; in this type of school mathematics in an important subject.

${ }^{4}$ Baulac, Y., Bellemain, F., Laborde, J.-M., 1988, Cabri-Géomètre, un logiciel d'aide à l'apprentissage de la géométrie. Logiciel et manuel d'utilisation, Paris: Cedic-Nathan. The most relevant feature of Cabri from the didactical point of view is the dragging function, that is the possibility of directly manipulating the constructed figures on the screen: if a figure has been correctly drawn, according to the rules and properties of geometry, it keeps all its internal relationships whenever it undertakes dragging. Otherwise it messes up.

${ }^{5}$ For the references on which Imai's background is based see [33].
} 


\section{References}

1. Bartolini Bussi, M., Boero, P., Ferri, F., Garuti, R. and Mariotti, M. A., 1997, in Proceedings of PME 21, edited by E. Pekonen (Helsinki: University of Helsinki), 1, 180-195.

2. Chazan, D.: 1993, Educ. Studies Math., 24, 359-387.

3. Harel, G.\& Sowder, L., 1996, in Proceedings of PME 20, edited by A. Gutierrez \& L. Puig (Valencia: University of Valencia), 3, 59-66.

4. Healy, L. and Hoyles, C., 1998, Justifying and proving in school mathematics - Technical Report on the Nationwide Survey, Institute of Education, University of London.

5. Moore, R., 1994, Educ. Studies Math., 27, 249-266.

6. $\quad$ Lampert, M., 1990, Am. Educ. Res. J., 27 (1), 29-63.

7. Voigt, J., 1995, in The emergence of mathematical meaning: interaction in classroom cultures, edited by P. Cobb and H. Bauersfeld, (Hillsdale, NJ: Erlbaum), 163-201.

8. Pehkonen, E., 1991, ZDM, 91 (1), 1-4.

9. NCSM, 1989, Math. Teacher, 82 (6), 470-474.

10. Evans, J., 1987, Math. School, 16 (1), 48-50.

11. Javorski, B., 1994, Investigating Mathematics Teaching, (London: The Falmer Press).

12. Boaler, J., 1998, J. Res. Math. Educ., 29 (1), 41-62.

13. Stacey, K., 1991, ZDM, 91 (1), 8-14.

14. Mason, J., 1991, ZDM, 91 (1), 14-19.

15. Arsac, G., Germain, G. and Mante, M., 1988, Problème Ouvert et Situation - Problème, (Villeurbanne: IREM).

16. Kilpatrick, J., 1987, Cognitive science and mathematics education, edited by A. Schoenfeld, (Mahwah, NJ: LEA), 123-147.

17. Silver, E. A., 1994, For Learn. Math., 14 (1), 19-28.

18. Arzarello, F., Micheletti, C., Olivero, F., Paola, D. and Robutti, O., 1998, in Proceedings of $P M E$ 22, edited by A. Olivier and K. Newstead (Stellenbosch: University of Stellenbosch), 2, 24-31.

19. Arzarello, F., Gallino, G., Micheletti, C., Olivero, F., Paola, D. and Robutti, O., 1998, in Proceedings of PME 22, edited by A. Olivier and K. Newstead (Stellenbosch: University of Stellenbosch), 2, 32-39.

20. Brodie, K., 2000, For Learn. Math., 20 (1), 9-16.

21. Cobb, P., 1995, The emergence of mathematical meaning: interaction in classroom cultures (Hillsdale, NJ: Lawrence Erlbaum), 25-129.

22. Healy, L., Pozzi, S. and Hoyles, C., 1995, Cognition and instruction, 13 (4), 505-523.

23. Kieran, K. and Dreyfus, T., 1998, in Proceedings of PME 22, edited by A. Olivier and K. Newstead (Stellenbosch: University of Stellenbosch), 3, 112-119.

24. Edwards, J.-A. and Jones, K., 1999, in Proceedings of PME 23, edited by O. Zaslavski (Haifa: Technion), 2, 281-288.

25. Godino, J. D. and Batanero, C., 1994, Rech. didact. Math., 14 (3), 325-355.

26. Johnson, D. W., Johnson, R. T. and Holubec, E. J., 1994, The nuts and bolts of cooperative learning (Edina, MN: Interaction Book Company). 
27. Simon, M. A., 1996, Educ. Studies Math., 30, 197-210.

28. Zazkis, R., Dubinsky, E. and Dautermann, J., 1996, J. Res. Math. Educ., 27, 435-457.

29. Hintikka, J. and Remes, U., 1974, The method of analysis (Dordrecht/ Boston: Reidel).

30. Furinghetti, F. and Paola, D., 1998, in Proceedings of PME 22, edited by A. Olivier and K. Newstead (Stellenbosch: University of Stellenbosch), 2, 313-320.

31. Harel, G. and Tall, D., 1990, For Learn. Math., 11 (1), 38-42.

32. Kleiner, I. and Movshovitz-Hadar, N., 1997, Math. Intell., 19 (3), 16-26.

33. Imai, T., 2000, Int. J. Math. Educ. Sci. Technol., 31, 187-193.

34. Cobb, P., Yackel, E. and Wood, T, 1989, in Affects and mathematical problem solving, edited by D. B. McLeod \& V. M. Adams, (New York: Springer-Verlag), 117-148.

35. Yackel, E. and Cobb, P., 1996, J. Res. Math. Educ., 27, 458-477.

36. McLeod, D. B., 1989, in Affects and mathematical problem solving, edited by D. B. McLeod \& V. M. Adams, (New York: Springer-Verlag), 245-258.

37. McLeod., D. B., 1992, in Handbook of research on mathematics learning and teaching, edited by D. A. Grouws, (New York: Macmillan), 127-146. 